\title{
Wann ist eine Implantatentfernung nach Wirbelsäulenstabilisierung sinnvoll?
}

\author{
Isabel Graul, Ulrich Spiegl, Christoph Josten
}

\section{Zusammenfassung}

Bei der Vielzahl an Verletzungen der Wirbelsäule muss die Art und Höhe der Verletzung sowie die Anzahl der eingebundenen Segmente berücksichtigt werden. Eine Implantatentfernung an der BWS und LWS ist nach 12 Monaten und gesicherter ossärer Konsolidierung anzustreben. Im Falle von dorsoventralen Versorgungen, wobei die dorsale Stabilisierung unversehrte Segmente überbrückt, kann eine Materialentfernung bei sicherer ventraler Fusion ab dem 6. Monat angestrebt werden. Bei zusätzlicher additiver ventraler Platte/Stab ist bereits bei partieller Konsolidierung eine frühzeitige Materialentfernung nach 4 Monaten möglich. Bei dorsoventral-bisegmentaler Versorgung sollte die Indikation der Implantatentfernung kritisch gestellt werden. Ebenfalls sollte die Indikation zur Implantatentfernung bei degenerativen Erkrankungen kritisch abgewogen werden und z. B. nach vorheriger Infiltration mit Lokalanästhetikum zur Beurteilung der Schmerzreduktion erfolgen.

\section{When is it Useful to Remove Implants after Stabilising the Spine?}

Treatment of injuries to the spinal column must depend on the type and level of the disease, as well as the number of bridged segments. Dorsal, ventral, and combined dorsoventral approaches have been described. The procedures may be short segmental or use multilevel instrumentation. Moreover, dorsal instrumentation can be performed with or without dorsolateral spondylodesis. Thus, the indication for implant removal has to be made individually. Generally, dorsal implant removal in trauma patients can be performed after bony consolidation and about 12 months after surgery. In patients treated by a combined dorsoventral approach with bridging posterior instrumentation, earlier implant removal can be performed, after 6 to 9 months. This can be done already after the fourth month after surgery if an additional ventral plate was used. In contrast, implant removal after bisegmental dorsoventral spondylodesis is only indicated in cases of implant-associated pain or patient request. Similarly, implant removal should be discussed critically in patients with degenerative pathologies. Prior infiltration under local anesthesia is advisable.

\section{Einleitung}

In den meisten Fällen, in denen Osteosynthesematerial eingebracht wird, ist die Diskussion um eine Materialentfernung gegeben. Die Indikation hierzu wird oft kontrovers diskutiert. Die Gründe für operative Interventionen an der

OP-JOURNAL 2016; 32: 126-129

(C) Georg Thieme Verlag KG Stuttgart · New York DOI http://dx.doi.org/10.1055/s-0042-107043

der Wirbelsäule mit ihren eigenen Bewegungsfreiheiten, anatomischen Besonderheiten sowie biomechanischer Beanspruchung und der unterschiedlichen Verletzungen ist die Wirbelsäulenverletzung heterogen. Allerdings ist allen Versorgungen mit dorsaler Instrumentierung eines gemeinsam: Bewegungsabschnitte der Wirbelsäule werden zur Ausheilung der Verletzung ruhiggestellt. Bei der dorsalen Stabilisierung ohne Fusionierung kann nach Ausheilung der ossären Verletzung eine Materialentfernung erfolgen. Zudem kann nach knöchern konsolidierter Spondylodese die Implantatentfernung erfolgen. Meist sind nicht betroffene Segmente in die dorsale Stabilisierung eingebunden und können damit wieder freigegeben werden. Gerade an der Brustwirbelsäule sind zur Stabilisierung von Frakturen meist langstreckige Instrumentierungen, die in der Regel 4 oder mehr Bewegungssegmente mit einschließen, indiziert [1]. Somit werden auch nicht verletzte Bewegungssegmente überbrückt. Dies führt z.T. zu relevanten Einschränkungen der Beweglichkeit. So können erhebliche Limitationen bei Alltagsaufgaben entstehen. Insbesondere junge Patienten nach traumatischen Frakturen, welche noch im Arbeitsleben stehen, können hierdurch oft eingeschränkt sein. Hinzu kommt das Auftreten von Implantatversagen bei fehlender Spondylodese, die nicht selten nach 2-3 Jahren nach Implantation anzutreffen sind. Die Heterogenität der Wirbelsäulenverletzung und damit die Indikation zur Implantatentfernung ist in der derzeitigen Studienlage mit entsprechender Fallzahl nur gering abgebildet und klare Richtlinien lassen sich hieraus schwer ableiten.

\section{Vor- und Nachteile der Implantat- entfernung an der Wirbelsäule}

Die Hauptindikationen einer Implantatentfernung ist die Freigabe von Bewegungssegmenten zur Verbesserung der Funktion, implantatassoziierte Schmer- 
zen, Reduktion von Anschlussdegenerationen an der Wirbelsäule und das Verhindern von Materialversagen, z.B. Brüche der Längsträger. Durch eine Entfernung des Osteosynthesematerials an der Wirbelsäule können Bewegungsausmaße gesteigert werden sowie Reibekorrosion der metallenen Komponenten, allergische Reaktionen, Infektionen oder eine durch „Stress Shielding“ entstandene Osteopenie gemindert werden. Die Entfernung des internen Fixateurs kann durch die verminderte Steifheit des betroffenen Segments der Wirbelsäule die Stresskonzentration der angrenzenden Segmente verringern und so die Gefahr von Anschlussfrakturen und Anschlussdegenerationen senken. Weiterhin können durch die Entfernung Implantataffektionen aufgrund der paravertebralen Lage in der Muskulatur reduziert werden. Insbesondere sehr schlanke Patienten klagen häufig über implantatassozierte Beschwerden in Rückenlage und beim Sitzen, die durch eine Materialentfernung behoben werden können. Trotz der Vorteile ist die Implantatentfernung an der Wirbelsäule ein operativer Eingriff, der in Bauchlage durchgeführt wird mit den entsprechenden Risiken. Zu den speziellen Risiken dieser Operation zählen selten auftretende Infektionen in diesem Bereich, neurovaskuläre Verletzungen [2] und das Auftreten erneuter Schmerzen und eine kyphotische Nachsinterung mit Zunahme von Fehlstellungen [3].

\section{Traumatische Frakturen}

An der oberen BWS zeigen sich die Verletzungen oft ohne hochgradige Zerstörung des Wirbelkörpers. Zudem liegt eine geringere mechanische Belastung vor. Insbesondere der Brustkorb dient dabei als relevanter Stabilisator. Daneben muss auch die zugangsassoziierte Morbidität, insbesondere für die obere und mittlere BWS, bedacht werden, sodass häufig auf eine ventrale Stabilisierung verzichtet werden kann [4]. Meist sind jedoch langstreckige Versorgungen über 4 Bewegungssegmente indiziert. Insgesamt ist bei isoliert dorsaler Stabilisierung und insbesondere bei längerstreckiger Versorgung bei radiologisch gesicherter ossärer Konsolidierung eine Materialentfernung an der Brust- und Lendenwirbelsäule nach 12 Monaten anzustreben.

Die häufigsten Frakturen der Wirbelsäule sind mit $50 \%$ am thorakolumbalen Übergang, Th XI bis L II, beschrieben. Die einfachen A1-Frakturen werden meist einer konservativen Therapie zugeführt. Die Therapiestrategie ist jedoch individuell anzupassen, in Abhängigkeit von dem Habitus des Patienten, der Knochenqualität und dem Ausmaß der Bandscheibenläsion. Berstungsfrakturen, Distraktionsfrakturen und Torsionsfrakturen werden meist zunächst von dorsal reponiert und stabilisiert. Im thorakolumbalen Übergang und im lumbalen Bereich ist meist eine kurzstreckige Versorgung ausreichend. Abhängig von der Instabilität der Fraktur muss eine additive ventrale Versorgung erfolgen. Bei Vorhandensein von neurologischen Symptomen erfolgt die zusätzliche Dekompression, wodurch die lasttragende Wirbelsäule zusätzlich geschwächt wird, sodass die Indikation zur additiven ventralen Versorgung großzügiger gestellt werden muss [5].

Zur Abwägung, ob ein rein dorsales Vorgehen bei Frakturen an der throrakolumbalen Wirbelsäule ausreicht, sollte ein MRT der Region zur Beurteilung der Bandscheibensituation erfolgen. Bei unauffälliger Bandscheibensituation und fehlender Nachsinterung ist eine rein dorsale Stabilisierung ausreichend, sodass nach Sicherstellung der ossären Konsolidierung, die Implantatentfernung anzustreben ist. Die überbrückten Segmente zeigen in den Untersuchungen von Spiegl et al. [7] keine Minderung der Bandscheibenfachhöhe oder -funktion. Dabei konnte nachgewiesen werden, dass die Bandscheibenfachhöhe im Falle einer Frakturbeteiligung nach Entfernung der dorsalen Instrumentierung nach 12 Monaten besser erhalten bleiben kann, als dies nach früzeitigerer Implantatentfernung der Fall ist. Die Autoren stellten die Hypothese auf, dass die notwendigen Reparaturvorgänge sich über 12 Monate erstrecken und somit die Materialentfernung bei isoliert dorsalem Vorgehen bis nach dem 12. Monat hinausgezögert werden sollte.

Bei alleiniger dorsaler Stabilisierung und radiologisch gesicherter ossärer Konsolidierung ist eine Materialentfernung an der BWS, TLÜ und LWS nach 12 Monaten anzustreben.

Risikofaktoren für einen Repositionsverlust stellen die Verwendung von polyaxialen Implantaten, kurzstreckige Versorgungen ab dem 60 . Lebensjahr ohne Zementaugmentation und die Notwendigkeit von Laminektomien dar. Insgesamt konnte in 36\% unserer Patienten auf eine additive ventrale Stabilisierung verzichtet werden, sodass nach Materialentfernung alle Bewegungssegmente erhalten werden [6].

Erfolgt eine dorsoventrale Versorgung mit dorsal-bisegmentaler und ventralmonosegmentaler Versorgung, kann nach knöcherner ventraler Fusion im CT, üblicherweise nach dem 6. bis 9. Monat, die Materialentfernung initiiert werden (Abb. 1). Bei Einbringen von zusätzlichem ventralem Ostesosynthesematerial (Platte/Stab) ist die Materialentfernung der dorsalen Instrumentierung bereits nach partieller Konsolidierung zeitiger möglich. Bisegmentale dorsale und ventrale Versorgungen sollten lediglich dann angestrebt werden, falls implantatassoziierte Beschwerden vorliegen bzw. bei entsprechendem Patientenwunsch.

Bei einer von dorsoventral versorgten Fraktur mit längerstreckiger dorsaler Instrumentierung kann eine Verkürzung der Instrumentierung bereits ab dem 4. Monat zur Freigabe der unversehrten überbrückten Bewegungssegmente erfolgen (Abb. 2).

Jentzsch et al. 2015 [8] zeigten in 137 Patienten mit traumatischen Frakturen der Lendenwirbelsäule und des thorakolumbalen Übergangs eine Verbesserung der Schmerzempfindung und eine gleichzeitig verbesserte Funktion der dorsalen Instrumentierung nach Implantatentfernung bei dokumentierter Fusion. Im Durchschnitt wurde die Entfernung der dorsalen Instrumentierung nach 13 Monaten durchgeführt. Hier konnte eine nicht signifikante Verringerung des Cobb-Winkels von $0,1^{\circ}$ nach Materialentfernung gezeigt werden. Es bestand kein Unterschied zwischen initial posterior oder kombinierter operativer Versorgung in den postoperativen Ergebnissen nach Materialentfernung. Des Weiteren zeigte auch die Größe eines verwendeten Cages keinen Einfluss auf das Ergebnis. Die Schmerzsymptomatik, gemessen nach modifizierter Numerischer Rating-Skala (NRS), wurde zwar signifikant, jedoch klinisch nur unwesentlich um 0,5 reduziert. Dagegen konnten Jeon et al. [9] eine signifikante Schmerzreduktion von 1,6 Punkten in der visuellen Analogskala nach Implantatentfernung bei Berstungsfrakturen an der thorakolumbalen Wirbelsäule und primär rein dorsaler Versorgung nachweisen. Dabei erfolgte die Implantatentfernung nach 


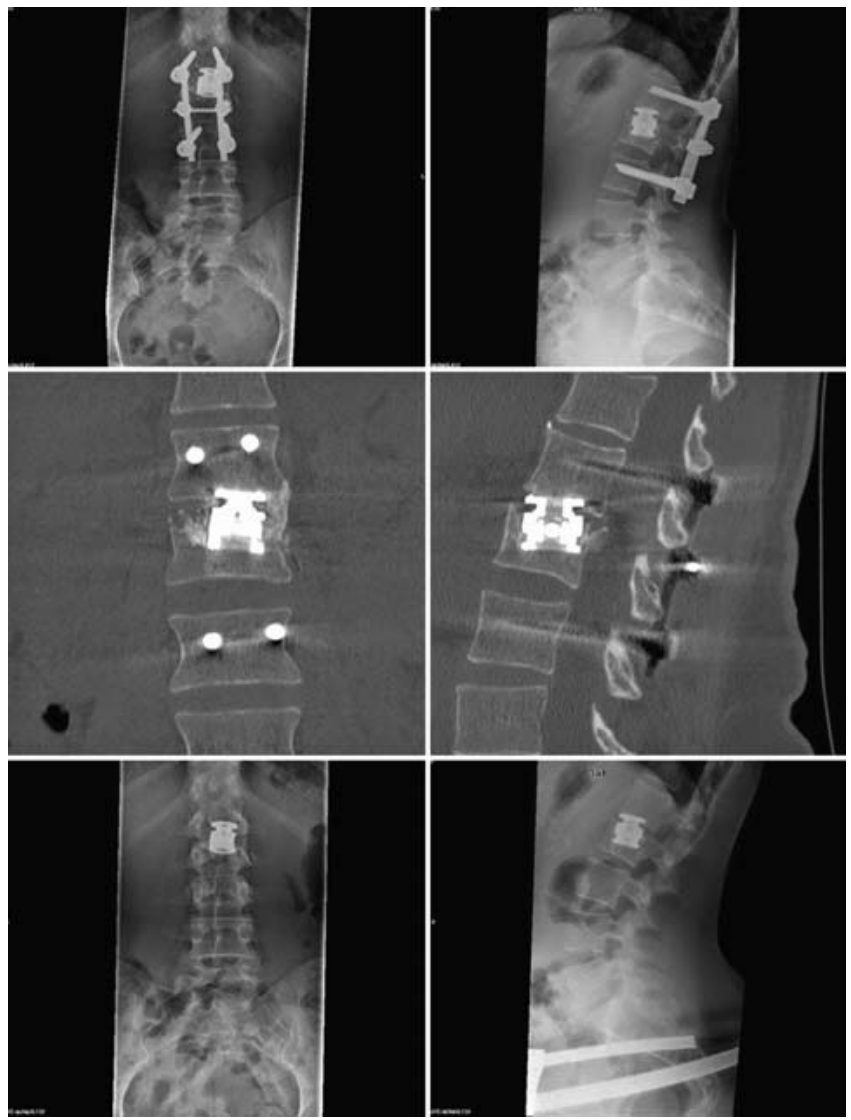

Abb. 1 Fallbeispiel: bisegmentale dorsoventrale Stabilisierung am thorakolumbalen Übergang. Eine junge Patientin stürzte von einer Leiter und zog sich eine LWK-II-Berstungsfraktur zu, es erfolgte die dorsale bisegmentale Stabilisierung von LWK I auf LWK III und eine zweizeitige anteriore monosegmentale Stabilisierung mit Cage-Einlage. Im CT nach 6 Monaten zeigt sich eine Fusion, sodass die Entfernung des dorsalen Fixateur interne nach 6 Monaten erfolgen konnte. Im weiteren Verlauf bestanden keine Schmerzen bei guter Beweglichkeit.
Fällen zeigen, dass 30 Patienten persistierende Schmerzen nach Implantatentfernung bei degenerativen lumbalen Eingriffen behielten. Hingegen konnten Alanay et al. [12] eine Verbesserung der Schmerzsymptomatik von $50 \%$, gemessen mittels NRS, und funktionelle Verbesserung in $84 \%$ in diesem Patientengut nachweisen.

Empfehlenswert ist bei irritierendem Material im Vorfeld jedoch eine Austestung der Schmerzintensität durch das Implantat mittels Infiltration durchzuführen. Daneben ist eine kritische und ausführliche Aufklärung des Patienten, insbesondere über die Möglichkeit verbleibender oder gar zunehmender Beschwerden nach Materialentfernung, anzuraten.

Bei einer isolierten dorsalen Dekompression ohne Fusion mit Instrumentierung ist bei einer Materialentfernung das Risiko der Erzeugung von Instabilitäten gegeben, sodass die Indikation zur Entfernung der Instrumentierung nur unter Vorbehalt und expliziter Aufklärung des Risikos erfolgen sollte.

\section{Diskussion}

Die Indikation zur Implantatentfernung muss kritisch diskutiert werden. In Abwägung der Risiken des operativen Eingriffs muss eine ausführliche Aufklärung des Patienten erfolgen.

In den Studien konnte insbesondere eine Indikation zur Implantatentfernung nach Konsolidierung bei aufgetretenen Mikrobewegungen, Reibeerosionen, allergischen Reaktionen, Infektionen, Stressspitzen auf die anliegenden Segmente
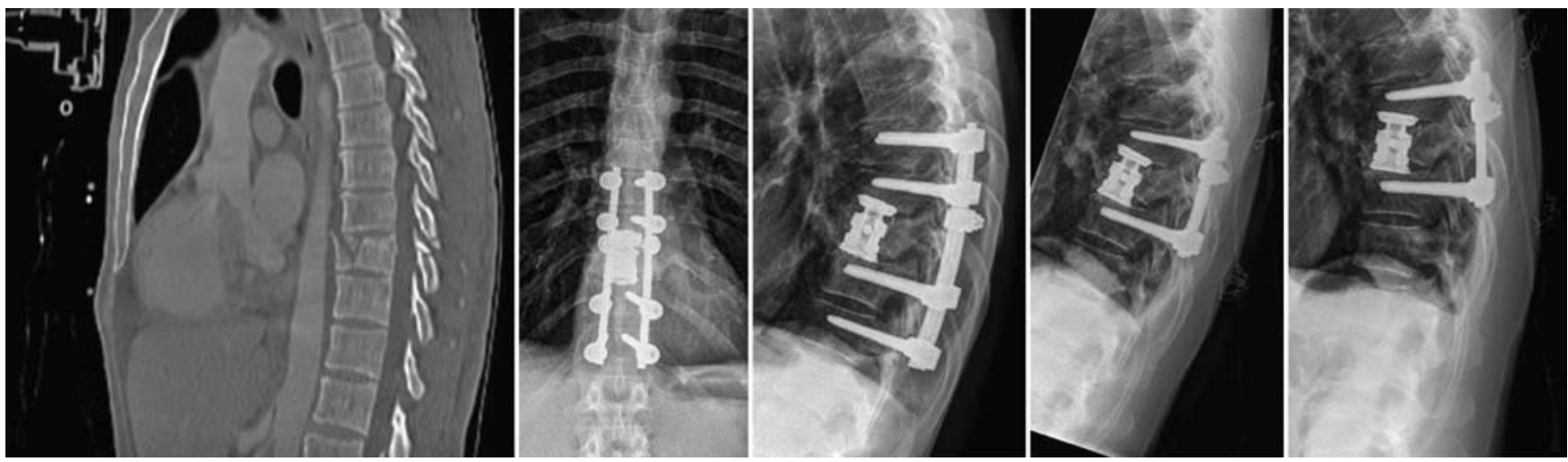

Abb. 2 Fallbeispiel: langstreckige dorsoventrale Stabilisierung der Brustwirbelsäule. Ein 40-jähriger Patient verunglückte bei einem Verkehrsunfall und wurde polytraumatisiert zuverlegt. Es erfolgte bei bestehender BWK-X-Fraktur die operative Versorgung mit dorsaler Stabilisierung von BWK VIII auf BWK XII sowie die anteriore Stabilisierung mit bisegmentalem expandierbarem Titancage und Spongiosaeinlage. Nach gesicherter knöcherner Durchbauung erfolgte die Freigabe der nicht betroffenen Segmente mit Teilentfernung des Fixateur externe auf eine dorsale Stabilisierung BWK IX auf BWK XI nach 12 Monaten. 

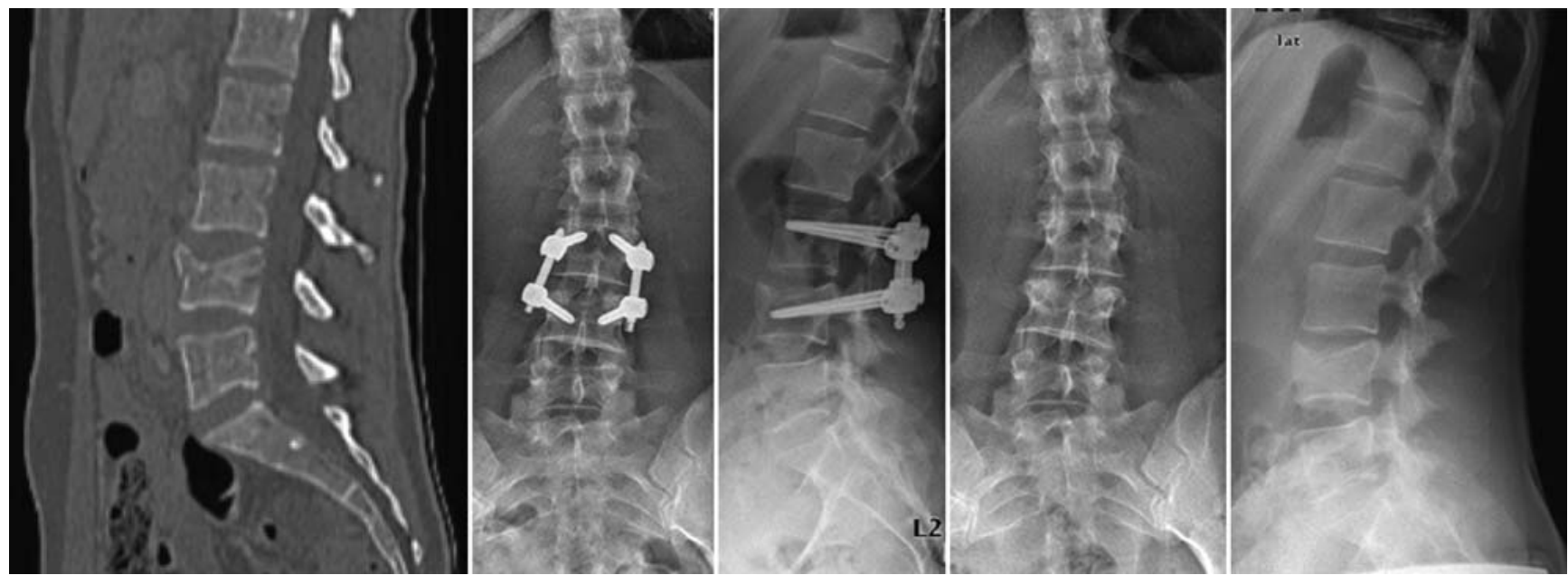

Abb. 3 Fallbeispiel: dorsale monosegmentale Stabilisierung der Lendenwirbelsäule. Die junge Patientin wurde polytraumatisiert nach einem Verkehrsunfall als Fahrradfahrerin u. a. mit einer Kompressionsfraktur des LWK IV in unserer Klinik vorgestellt. Es erfolgte die monosegmentale dorsale Stabilisierung der Fraktur. Nach 6 Monaten konnte die Implantatentfernung erfolgen. Es zeigte sich nur eine geringgradige Minderung der Höhe des Bandscheibenfachs L III/L IV. Subjektiv klagte die Patientin über keinerlei Beschwerden und zeigte eine sehr gute Funktion.

oder Fehllagen der Schrauben und Implantatversagen gesehen werden.

Weiterhin sollte die mit der dorsalen Instrumentierung erworbene Bewegungseinschränkung bei der Entscheidungsfindung eine Rolle spielen. Auch der implantatassozierte Dyskomfort durch die paravertebrale Lage in der Muskulatur kann bei sicher nachgewiesener Assoziation durch Umflutung der Schraubensysteme mit Lokalanästhetikum eine ausschlaggebende Rolle spielen. Aber gerade bei verbliebenen Schmerzen nach Wirbelsäuleneingriffen sollte kritisch hinterfragt werden, ob eine Beschwerdebesserung nach Implantatentfernung zu erwarten ist. Es zeigt sich allerdings häufig eine Verbesserung der Funktion sowie teilweise der Schmerzempfindung nach traumatischen Verletzungen. Bei den degenerativen Erkrankungen ist die Indikation weiter kontrovers zu diskutieren. Die Komplikationsrate von 3-9\% [2] sollte hierbei mit berücksichtigt werden. In diesen Fällen empfiehlt sich die Abklärung der Ursachen der Schmerzsymptomatik wie Lockerung, Pseudarthrose, Low-grade-Infektion oder Abrieb.

Bei der Indikationsstellung zur Implantatentfernung muss auf das Risiko einer Nachsinterung mit kyphotischer Fehlstellung hingewiesen werden, welche erneut zu Bewegungseinschränkungen und Schmerzen führen kann. In solchen Fällen kann eine Re-Instrumentierung nötig werden.

\section{Fazit}

Insbesondere bei jüngeren Patienten sehen wir den Vorteil in der Freigabe von Bewegungssegmenten zur Verbesserung der Funktion und Reduktion von Anschlussdegenerationen an der Wirbelsäule. Deshalb besteht mehrheitlich die Indikation zur Implantatentfernung nach traumatischer Wirbelsäulenverletzung und sichergestellter ossärer Konsolidierung.

Bei älteren Patienten mit degenerativen Wirbelsäulenverletzungen erachten wir bei belegten implantatassoziierten Schmerzen und konsolidierter Fusion die Indikation zur Implantatentfernung nach ausführlicher Aufklärung der Patienten als gegeben.

\section{Literatur}

1 Josten C, Katscher S, Gonschorek O. [Treatment concepts for fractures of the thoracolumbar junction and lumbar spine]. Orthopade 2005; 34: 1021-1032

${ }^{2}$ Krettek C, Müller C, Meller R et al. [Is routine implant removal after trauma surgery sensible?] Unfallchirurg 2012; 115: 315-322

3 Spiegl U, Hauck S, Merkel P et al. Six-year outcome of thoracoscopic ventral spondylodesis after unstable incomplete cranial burst fractures of the thoracolumbar junction: ventral versus dorso-ventral strategy. Int Orthop 2013; 37: 1113-1120

${ }^{4}$ Katscher S, Verheyden P, Gonschorek $O$ et al. [Thoracolumbar spine fractures after conservative and surgical treatment. Dependence of correction loss on fracture level]. Unfallchirurg 2003; 106: 20-27

5 Josten C, von Bühren V. Die verletzte Wirbelsäule. Berlin, Heidelberg: Springer; 2013

${ }^{6}$ Spiegl U, Jarvers JS, Heyde CE et al. Zeitverzögerte Indikationsstellung zur additiv ventralen Versorgung traumatischer thorakolumbaler Berstungsfrakturen nach primär dorsaler Stabilisierung. Welchen Korrekurverlust erwarten wir? Unfallchirurg 2015; 113: $1-9$

${ }^{7}$ Spiegl U, Jarvers JS, Glasmacher S et al. Freigabe von Bewegungssegmenten nach dorsaler Stabilisierung. Unfallchirurg 2014; 113: 1-8

8 Jentzsch T, Gomes de Lima V, Seifert B et al. The benefits of elective spinal implant removal: a retrospective study of 137 patients. Eur Spine J 2016; 25: 856-864

9 Jeon CH, Lee HD, Lee YS et al. Is it benifical to remove the pedicle screw instrument after successful posterior fusion of thoracolumbar burst fractures? Spine (Phila Pa 1976) 2015; 40: E627-E633

${ }^{10}$ Reinhold M, Knop C, Beisse R et al. Operative treatment of 733 patients with acute thoracolumbar spinal injuries: comprehensive results from the second, prospective, internetbased multicenter study of the Spine Study Group of the German Association of Trauma Surgery. Eur Spine J 2010; 19: 1657-1976

11 Hume M, Capen DA, Nelson RW. Outcome after Wiltse pedicle screw removal. J Spinal Disord 1996; 9: 121-124

${ }^{12}$ Alanay A, Vyas R, Shamie AN. Safety and efficacy of implant removal for patients with recurrent back pain after a failed degenerative lumbar spine surgery. J Spinal Disord Tech 2007; 20: 271-277

\section{Dr. med. Isabel Graul \\ Assistenzärztin \\ PD Dr. med. Ulrich Spiegl \\ Oberarzt \\ Prof. Dr. med. Christoph Josten \\ Geschäftsführender Direktor \\ Klinik und Poliklinik für \\ Unfallchirurgie, Orthopädie \\ und plastische Chirurgie \\ Universitätsklinik Leipzig \\ Liebigstraße 20 \\ 04103 Leipzig}

UlrichJosefAlbert.Spiegl@

medizin.uni-leipzig.de 